\title{
An Approximate Technique for Solving Lagerstrom Equation
}

\author{
Md. Zahangir Alam*, Md. Shamsul Alam², Md. Nazmul Sharif ${ }^{3}$
}

\begin{abstract}
The Lagerstrom's equation has been solved by an approximate technique combining both homotopy perturbation and variational iteration method. By this technique the solution of Lagerstrom's equation can be determined for viscous flow past a solid at low Reynolds number where a significance mater is the occurrence of logarithmic term. In this technique ExplntegralEi function has been used for simplifying the calculation. The results have been calculated by this technique shows a good agreement with those obtained by numerical method.

Keywords: ExplntegralEi, Homotopy perturbation method, Lagerstrom's equation.

2010 AMS: Primary 34B15, 34B16, 34E15, Secondary

${ }^{1}$ Department of Mathematics, Rajshahi University of Engineering \& technology (RUET), Rajshahi, Bangladesh, ORCID: 0000-0003-0128-6920

2 Department of Mathematics, Rajshahi University of Engineering \& technology (RUET), Rajshahi, Bangladesh, ORCID: 0000-0002-6325-6797

${ }^{3}$ Department of Mathematics, Rajshahi University of Engineering \& technology (RUET), Rajshahi, Bangladesh, ORCID: 0000-0003-4234-6651

*Corresponding author: zahangiramth@gmail.com

Received: 1 April 2020, Accepted: 24 June 2020, Available online: 30 June 2020
\end{abstract}

\section{Introduction}

The perturbation methods [1]-[2] are widely used for solving nonlinear problems mostly in week nonlinear problems. For Lagerstrom's equation a logarithmic singularity arises and the straight forward perturbation method fails to give uniformly valid solution. Several methods are used to solve Lagerstrom's model equation. Earlier P. A. Lagerstrom [3] used Matched asymptotic expansions. A geometric analysis and Rigorous asymptotic expansion methods have introduced by N. Popovic, P. Szmolyan [4]-[5]. S. Kaplun and P. A. Lagerstrom [6] have presented asymptotic expansions of Navier-Stokes solutions for small Reynolds numbers. N. Fenichel [7], K. K. Alymkulov and D. A. Tursunov [8], P. A. Lagerstrom and R. G. Casten [9] used singular perturbation technique for solving ordinary differential equation. Lagerstrom's model equation is given by the non-autonomous second-order boundary value problem

$$
u^{\prime \prime}+\frac{n-1}{x} u^{\prime}+u u^{\prime}=0,
$$

where

$$
u(\varepsilon)=0, u(\infty)=1,
$$

with $n \in N, 0<\varepsilon \leq \infty$ and prime denotes differentiating with respect to $x$. 
Let $\xi=\frac{x}{\varepsilon}$ then Eq. (1.1) and Eq. (1.2) can be written as

$$
u^{\prime \prime}+\frac{n-1}{\xi} u^{\prime}+\varepsilon u u^{\prime}=0,
$$

and

$$
u(1)=0, u(\infty)=1,
$$

with $1<\xi<\infty$ and prime denotes differentiating with respect to $\xi$. Eq. (1.1) and Eq. (1.3) are the model which was first introduce in [6] and [10] for viscous flow past a solid at low Reynolds number. When $\varepsilon=0$ the solution of Eq. (1.3) becomes $u=1-\frac{1}{\xi}$ and it is known as unperturbed solution. In this paper a new technique has been presented to solve Eq. (1.3) with boundary conditions in equation Eq. (1.4) based on combined homotopy perturbation method [11] and variational iteration method [12].

\section{The Method}

First we consider the Lagerstrom's model equation is given in Eq. (1.3) as of the form

$$
u^{\prime \prime}+\frac{n-1}{\xi} u^{\prime}+k u^{\prime}+p(u-k) u^{\prime}=0,
$$

where

$$
u(1)=0, u(\infty)=1 .
$$

When $p=0$ Eq. (2.1) has a solution that one can be determined easily. When $p \leq 1$ we consider the approximate solution of the form [11]

$$
u=u_{0}+p u_{1}+p^{2} u_{2}+\mathscr{O}\left(p^{3}\right) .
$$

On substituting the value of $u$ in Eq. (2.1) we get

$$
u_{0}^{\prime \prime}+\frac{n-1}{\xi} u_{0}^{\prime}+k u_{0}^{\prime}+\left(u_{1}^{\prime \prime}+\frac{n-1}{\xi} u_{1}^{\prime}+k u_{1}^{\prime}+\left(u_{0}-k\right) u_{0}^{\prime}\right) p+\left(u_{2}^{\prime \prime}+\frac{n-1}{\xi} u_{2}^{\prime}+k u_{1}^{\prime}+\left(u_{0}-k\right) u_{1}^{\prime}+u_{1} u_{0}^{\prime}\right) p^{2}+\mathscr{O}\left(p^{3}\right)=0 .
$$

Equating the coefficient of like power of $p$ we obtain

$$
\begin{aligned}
& u_{0}^{\prime \prime}+\frac{n-1}{\xi} u_{0}^{\prime}+k u_{0}^{\prime}=0, \\
& u_{1}^{\prime \prime}+\frac{n-1}{\xi} u_{1}^{\prime}+k u_{1}^{\prime}+\left(u_{0}-k\right) u_{0}^{\prime}=0, \\
& u_{2}^{\prime \prime}+\frac{n-1}{\xi} u_{2}^{\prime}+k u_{1}^{\prime}+\left(u_{0}-k\right) u_{1}^{\prime}+u_{1} u_{0}^{\prime}=0 .
\end{aligned}
$$

Solving above set of linear equations we get $u_{0}, u_{1}, u_{2}, \cdots$, etc. and substituting these in Eq. (2.3) we obtain an approximate solution 


\section{Examples}

\subsection{Example 1}

Let us first consider $n=3$ then Eq. (2.5), Eq. (2.6) and Eq. (2.7) turned into the flowing form

$$
\begin{aligned}
& u_{0}^{\prime \prime}+\frac{2}{\xi} u_{0}^{\prime}+k u_{0}^{\prime}=0, \\
& u_{1}^{\prime \prime}+\frac{2}{\xi} u_{1}^{\prime}+k u_{1}^{\prime}+\left(u_{0}-k\right) u_{0}^{\prime}=0, \\
& u_{2}^{\prime \prime}+\frac{2}{\xi} u_{2}^{\prime}+k u_{1}^{\prime}+\left(u_{0}-k\right) u_{1}^{\prime}+u_{1} u_{0}^{\prime}=0 .
\end{aligned}
$$

Obviously Eq. (3.1) can be rewritten as

$$
\frac{d}{d \xi}\left(\xi^{2} e^{k \xi} u_{0}^{\prime}\right)=0
$$

where $\xi^{2} e^{k \xi}$ is an integrating factor of Eq. (3.1).

Integrating Eq. (3.4) with respect to $\xi$ we get

$$
u_{0}^{\prime}=\frac{B e^{-k \xi}}{\xi^{2}} .
$$

Again integrating Eq. (3.5) with respect to $\xi$ we get

$$
u_{0}=A+B\left(-\frac{e^{-k \xi}}{\xi}-k \text { ExpIntegralEi }(-k \xi)\right),
$$

where ExpIntegralE $i$ function is defined as

$$
E i(x)=\gamma+\ln x+\exp \left(\frac{x}{2}\right) \sum_{n=1}^{\infty} \frac{(-1)^{n-1} x^{n}}{n ! 2^{n-1}} \sum_{k=0}^{\frac{n-1}{2}} \frac{1}{2 k+1},
$$

and $\gamma$ is Euler Gama constant.

Using condition $u_{0}(1)=0$ and from Eq. (3.6) we obtain

$$
A+B\left(-e^{-k}-k E i(-k)\right)=0 .
$$

Since at $\xi \rightarrow \infty,-\frac{e^{-k \xi}}{\xi}-k E i(-k \xi) \rightarrow 0$ and for satisfying the condition $u_{0}(\infty)=1, A$ must be considered as 1 then solving Eq. (3.8) for $B$ we obtain

$$
B=\frac{e^{k}}{1+e^{k} k E i(-k)}
$$

Substituting the values of $A$ and $B$ in Eq. (3.6) we obtain

$$
u_{0}=1+\frac{e^{k}}{1+e^{k} k \operatorname{Ei}(-k)}\left(-\frac{e^{-k \xi}}{\xi}-k \text { ExpIntegralEi }(-k \xi)\right),
$$

where $k$ is constant to be determined. 
We consider $\int_{0}^{\infty}\left(u_{0}-k\right) u_{0}^{\prime} d \xi \cong 0$ and we have obtain $k \cong 0.5$. For solving $u_{1}^{\prime}$ Eq. (3.2) can be written as

$$
\frac{d}{d \xi}\left(\xi^{2} e^{k \xi} u_{1}^{\prime}\right)=\left(\xi^{2} e^{k \xi}\left(k-u_{0}\right) u_{0}^{\prime}\right)
$$

where $\xi^{2} e^{k \xi}$ is an integrating factor. Substituting the values of $u_{0}$ in Eq. (3.11) and then integrating we obtain

$$
\xi^{2} e^{k \xi} u_{1}^{\prime}=\frac{e^{k}\left(e^{k(1-\xi)}-\xi+k \xi+e^{k}(k-1) k \xi E i(-k)+e^{k}(1+k \xi) E i(-k \xi)\right)}{\left(1+e^{k} k E i(-k)\right)^{2}}+C
$$

Using condition $u_{1}^{\prime}(1)=0$ then from Eq. (3.12) we obtain

$$
C=-\frac{e^{k}\left(k+e^{k}(k-1) k E i(-k)+e^{k}(1+k) E i(-k)\right)}{\left(1+e^{k} k E i(-k)\right)^{2}} .
$$

Substituting the values of $C$ in Eq. (3.12) we obtain

$$
u_{1}^{\prime}=\frac{g}{\xi^{2} e^{k \xi}},
$$

where

$$
g=\frac{e^{k}\left(e^{k(1-\xi)}-\xi+k \xi+e^{k}(k-1) k \xi E i(-k)+e^{k}(1+k \xi) E i(-k \xi)\right)}{\left(1+e^{k} k E i(-k)\right)^{2}}-\frac{e^{k}\left(k+e^{k}(k-1) k E i(-k)+e^{k}(1+k) E i(-k)\right)}{\left(1+e^{k} k E i(-k)\right)^{2}} .
$$

We have calculated $u_{1}$ by numerical integration as

$$
u_{1}=\int_{1}^{\xi} \frac{g}{\xi^{2} e^{k \xi}} d \xi
$$

Thus the approximate solution can be written for $p=1$

$$
u=u_{0}+u_{1},
$$

where $u_{0}$ and $u_{1}$ are obtain from Eq. (3.10) and Eq. (3.16).

\subsection{Example 2}

Consider $n=2$ and proceeding in a similar way as example 1 then we obtain

$$
u_{0}=1-\frac{E i(-k \xi)}{E i(-k)}
$$

and

$$
u_{1}=\int_{1}^{\xi} \frac{f}{\xi e^{k \xi}} d \xi
$$

where

$$
f=\frac{e^{-k \xi}+k \xi(E i(-k)-k E i(-k)-E i(-k \xi))}{k E i(-k)^{2}}+\frac{e^{-k}+k E i(-k)^{2}}{k E i(-k)^{2}} .
$$

Thus the approximate solution can be written for $p=1$

$$
u=u_{0}+u_{1},
$$

where $u_{0}$ and $u_{1}$ are obtain from Eq. (3.18) and Eq. (3.19). 


\section{Results and Discussion}

In the present work a new technique has been presented for solving Lagerstrom's model equation based on combined homotopy perturbation method [11] and variational iteration method [12]. First the approximate solution obtained by present procedure by Eq. (3.17) compared with numerical solution for $n=3, k=0.544$ have presented in Table 1 also we have presented the absolute percentage error in Table 1. Next the approximate solution obtained by present procedure by Eq. (3.21) compared with numerical solution for $n=2, k=0.49$ have presented in Table 2 also we have presented the absolute percentage error in Table 2.

\begin{tabular}{|c|c|c|}
\hline$\xi$ & Numerical Result & Present Result \\
$\operatorname{Er}(\%)$ \\
\hline 1.00 & 0.00000 & 0.00000 \\
& & 0.000000 \\
\hline 1.25 & 0.376978 & 0.376613 \\
& & 0.096 \\
\hline 1.50 & 0.60695 & 0.606553 \\
& & 0.065 \\
\hline 1.75 & 0.748508 & 0.749424 \\
& & 0.122 \\
\hline 2.00 & 0.836696 & 0.839742 \\
& & 0.364 \\
\hline 2.25 & 0.892385 & 0.897609 \\
& & 0.585 \\
\hline 2.50 & 0.928041 & 0.935012 \\
& & 0.751 \\
\hline 2.75 & 0.951176 & 0.959282 \\
& & 0.852 \\
\hline 3.00 & 0.966376 & 0.975006 \\
& & 0.893 \\
\hline 3.50 & 0.983257 & 0.991504 \\
& & 0.838 \\
\hline 4.00 & 0.990994 & 0.997712 \\
& & 0.677 \\
\hline 4.50 & 0.994656 & 0.999413 \\
& & 0.478 \\
\hline & & \\
\hline & & \\
\hline
\end{tabular}

Table 1. Comparison between the numerical results and present results for $n=3, k=0.544$ and $\operatorname{Er}(\%)$ denote the absolute percentage error with numerical result. 


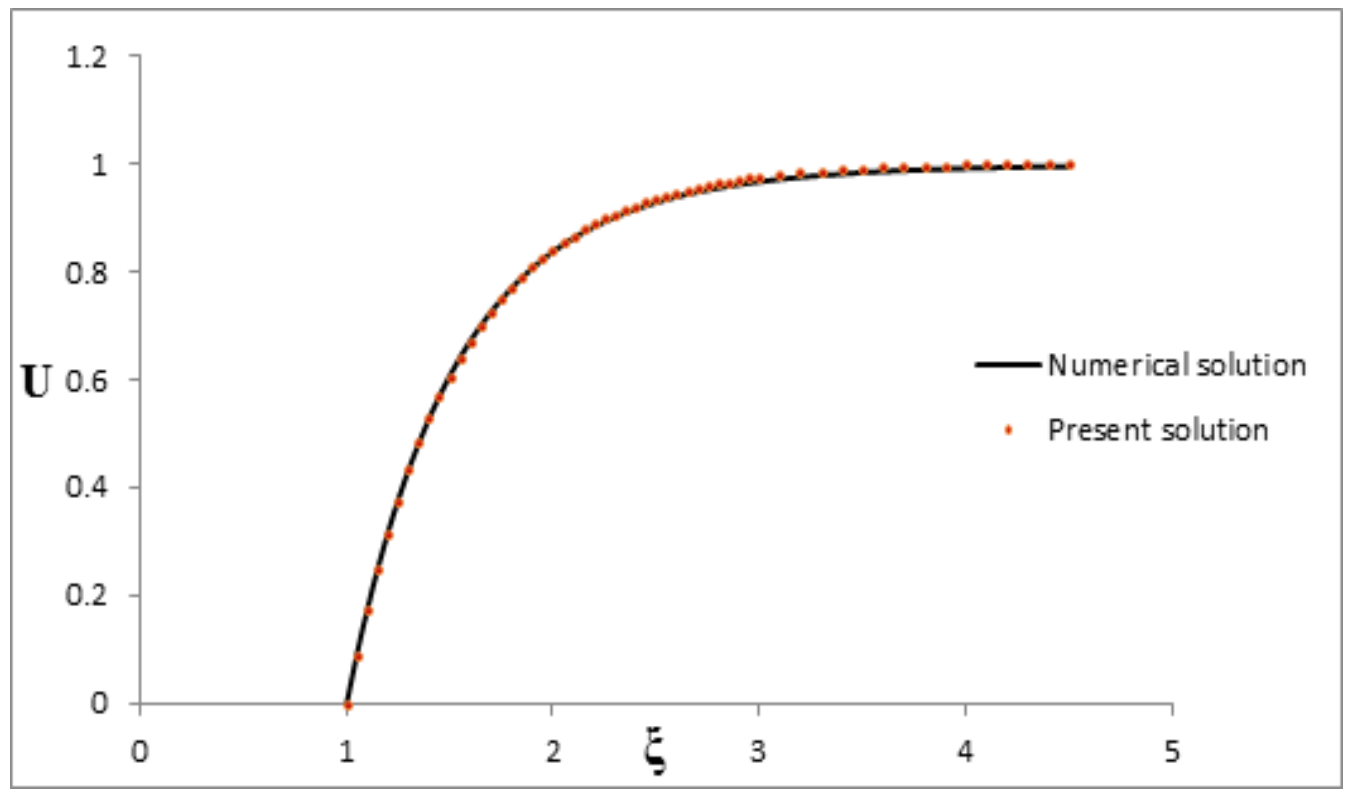

Figure 4.1. Present method solution has been presented by red colour circle line and black colour solid line represents numerical solution when $n=3, k=0.544$.

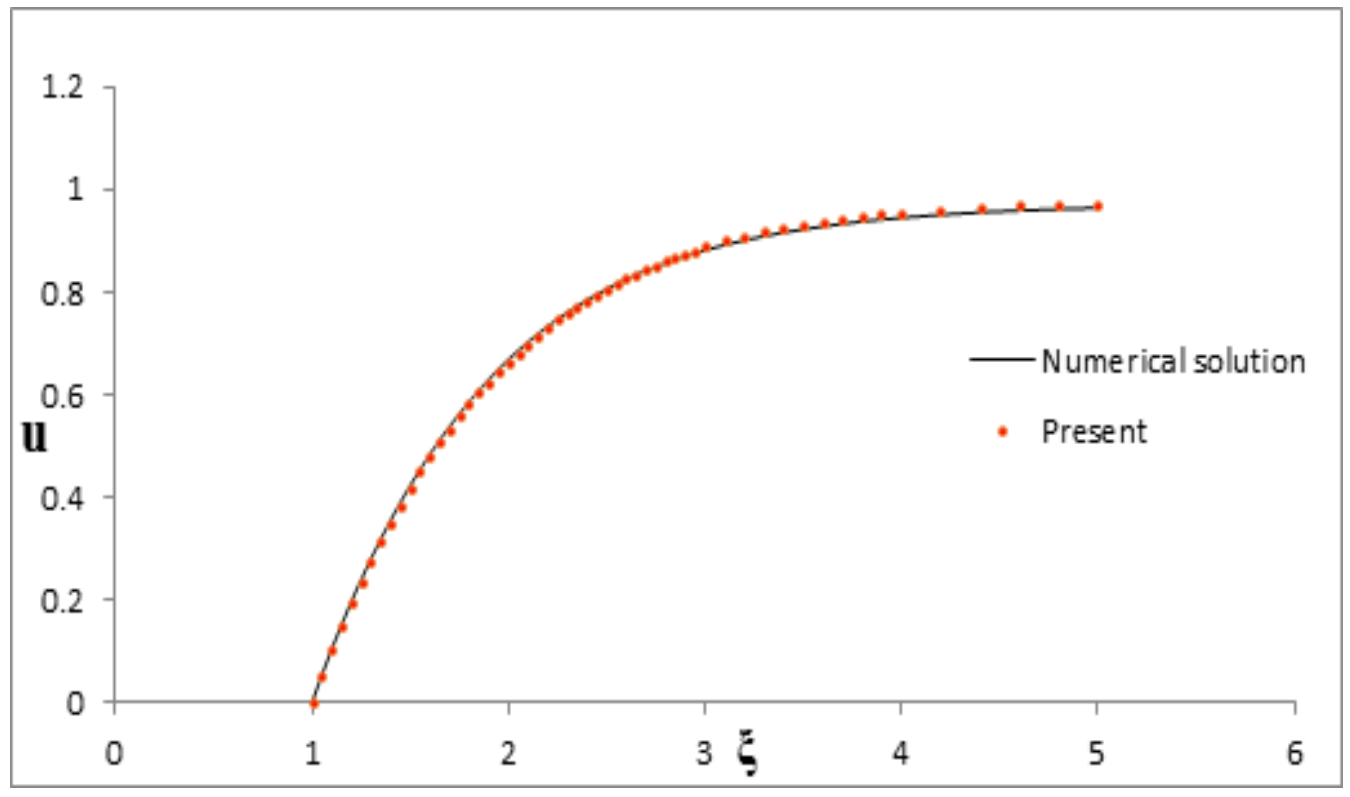

Figure 4.2. Present method solution has been presented by red colour circle line and black colour solid line represents numerical solution when $n=2, k=0.49$. 


\begin{tabular}{|c|c|c|}
\hline$\xi$ & Numerical Result & $\begin{array}{c}\text { Present Result } \\
E r(\%)\end{array}$ \\
\hline 1.00 & 0.00000 & $\begin{array}{c}0.00000 \\
0.000000\end{array}$ \\
\hline 1.25 & 0.23860 & $\begin{array}{c}0.23631 \\
0.959\end{array}$ \\
\hline 1.50 & 0.42265 & $\begin{array}{c}0.41829 \\
1.031\end{array}$ \\
\hline 1.75 & 0.56288 & $\begin{array}{c}0.55769 \\
0.922\end{array}$ \\
\hline 2.00 & 0.66853 & $\begin{array}{c}0.66414 \\
0.656\end{array}$ \\
\hline 2.25 & 0.74745 & $\begin{array}{c}0.74519 \\
0.302\end{array}$ \\
\hline 2.50 & 0.80605 & $\begin{array}{c}0.80667 \\
0.076\end{array}$ \\
\hline 2.75 & 0.84941 & $\begin{array}{c}0.85308 \\
0.432\end{array}$ \\
\hline 3.00 & 0.88143 & $\begin{array}{c}0.88788 \\
0.731\end{array}$ \\
\hline 3.50 & 0.92438 & $\begin{array}{c}0.93268 \\
0.898\end{array}$ \\
\hline 4.00 & 0.94681 & $\begin{array}{c}0.95595 \\
0.965\end{array}$ \\
\hline 4.50 & 0.95913 & $\begin{array}{c}0.96661 \\
0.779\end{array}$ \\
\hline 5.00 & 0.96595 & $\begin{array}{c}0.97006 \\
0.425\end{array}$ \\
\hline
\end{tabular}

Table 2. Comparison between the numerical results and present results for $n=2, k=0.49$ and $\operatorname{Er}(\%)$ denote the absolute percentage error with numerical result.

Then we have presented the numerical solution by black color solid line and present method solution by red color circle line for $n=3, k=0.544$ in figure 4.1 and in figure 4.2 we have presented the numerical solution by black color solid line and present method solution by red color circle line for $n=2, k=0.49$. We observe that in all tables and figure the present method solution shows a good coincide with numerical results.

\section{Conclusion}

A new method has been presented for solving Lagerstrom's equation having a significance mater is the occurrence of logarithmic term. The results obtained by the present paper are nicely shows a good agreement with corresponding numerical solutions for several values of $n$.The method is useful for solving nonlinear logarithmic singularity arising in science and engineering.

\section{References}

[1] A. H. Nayfeh, Perturbation Method, John Wiley \& Sons, New York, 1973.

[2] A. H. Nayfeh, D. T. Mook, Nonlinear oscillations, John Wiley \& Sons, New York, 1979.

[3] P. A. Lagerstrom, Matched asymptotic expansions: ideas and techniques, Applied Mathematical sciences, Springer-verlag, New York, 76, 1988.

[4] N. Popovic, P. Szmolyan, A geometric analysis of the Lagerstrom model problem, J. Diff. Eqs., 199(2) (2004), 290-325.

[5] N. Popovic, P. Szmolyan, Rigorous asymptotic expansions for Lagerstrom's model equation-a geometric approach, Nonlinear Analysis, 59 (2004), 531-565. 
[6] S. Kaplun, P.A. Lagerstrom, Asymptotic expansions of Navier-Stokes solutions for small Reynolds numbers, J. Math. Mech., 6 (1957), 585-593.

[7] N. Fenichel, Geometric Singular Perturbation Theory for Ordinary Differential Equations, J. Diff. Eqs., 31 (1979), 53-98.

[8] K. K. Alymkulov, D. A. Tursunov, Perturbed Differential Equations with Singular Points, Perturbation Theory, Dimo I, Uzunov, Intech Open, London, UK, 2017.

[9] P. A. Lagerstrom, R. G. Casten, Basic concepts underlying singular perturbation techniques, SIAM rev., 14(1) (1972), 63-120.

[10] P. A. Lagerstrom, A course on perturbation methods, Lecture Notes by M. Mortell, National University of Ireland, Cork, 1966.

[11] J. He, Homotopy perturbation method: A new nonlinear analytical technique, Applied Mathematics and Computational, 135 (2003), 73-79.

[12] J. He, Variational iteration method-Some recent results and new interpretations, Journal of Computational and applied Mathematics, 207 ( 2007), 3-17. 